\title{
Physical Activity to Stay Fit
}

\author{
Arkierupaia Shadap ${ }^{1}$ \\ ${ }^{1}$ Sikkim Manipal College of Nursing, Sikkim Manipal University, \\ Gangtok, Sikkim, India
}

\begin{abstract}
Address for correspondence Arkierupaia Shadap, MSc, Sikkim Manipal College of Nursing, Sikkim Manipal University, Gangtok, Sikkim 737102, India (e-mail: arkierupaiashadap.p@smims.smu.edu.in).
\end{abstract}

J Health Allied Sci ${ }^{\mathrm{Nu}: 2021 ; 11: 8-11}$

Abstract


Keywords
- physical activity
- physical movement
- exercise
- health

Physical activity is any bodily movement produced by skeletal muscles that require energy expenditure. According to World Health Organization, regular physical activities such as walking, cycling, doing the household work, etc., make an individual feel good and it has significant benefits for health. One of the vital elements for healthy bones at every age is exercise. Exercise lowers the blood pressure, slightly decreases the levels of total-and low-density lipoprotein-the bad cholesterol, and increases the levels of high-density lipoprotein-the good cholesterol. Regular physical activity and exercise help in maintaining the healthy body weight, decreasing stress, apprehension, and preventing depression. Exercising can also possibly help in delaying the effects of Alzheimer's disease and other forms of dementia. Strong evidence shows that physical activity has a wide range of beneficial effects for both physical and mental well-being.

\section{Introduction}

Physical activity is described as any physical movement produced by means of skeletal muscle mass that requires energy expenditure. The term "physical interest" is not equal to "exercise." ${ }^{1}$ According to World Health Organization, regular physical activities such as walking, cycling, doing the household work, etc., make an individual feel good and thus exercise has significant benefits for health. It reduces the risk of cardiovascular disease, diabetes and some cancers, helps control weight, and contributes to mental well-being. Being daily active has multiple benefits for all ages and especially for the growing age, i.e., children, young individual, and can make an extensive change to the health and welfare of senior population. On the contrary, physical inactivity and a sedentary lifestyle are among the leading risk factors for the health of the population. It is estimated to attribute to one million deaths per year in the WHO European Region. ${ }^{1}$ There are plenty of ways to make one-self engage in physical activity, be it at home, at work place, school, and transport area. All of us have choices at places where we live.

According to the National Institute of Health, 2018, one of the vital elements for healthy bones at every age is exercise. Exercise is important for treating and preventing osteoporosis. Muscle is a living tissue that responds to physical activity for getting stronger, and so does the bone. Therefore, published online

December 2, 2020
DOI https://doi.org/

$10.1055 / \mathrm{s}-0040-1721230$

ISSN 2582-4287. exercise improves the muscle strength, helps with coordination and balance, which eventually makes a healthy bone and lead to overall better health. The maximum bone density and strength are seen among young women and men who exercise regularly than those who do not. The majority of human bones start losing its bone density when they complete their third decade of life. Bone loss can be prevented among older women and men of age above 20 years with regular exercise. Exercising can help an individual maintain muscle power, dexterity, which in turn, helps to prevent falls and falls-related fractures. This is especially crucial for older adults and those who have been recognized with osteoporosis. ${ }^{2}$

\section{Benefits of Exercises}

Regular exercise is one of the best things to do for good health. It has countless benefits, including improving our overall health and fitness, and reducing the risk of many chronic diseases. ${ }^{3}$ Strong evidence shows that physical activity has a wide range of beneficial effects for both physical and mental well-being. ${ }^{1}$ Exercises decrease $25 \%$ risk of many disorders such as cardiovascular disease and type 2 diabetes. Substantially exercise reduces the risk of hypertension and some forms of cancer. Regular physical activity and exercise help in maintaining the healthy body weight, decreasing one's
This is an open access article published by Thieme under the terms of the Creative Commons Attribution-NonDerivative-NonCommercial-License, permitting copying and reproduction so long as the original work is given appropriate credit. Contents may not be used for commercial purposes, or adapted, remixed, transformed or built upon. (https://creativecommons.org/licenses/by-nc-nd/4.0/)

Thieme Medical and Scientific Publishers Pvt. Ltd., A-12, 2nd Floor, Sector 2, Noida-201301 UP, India 
stress, apprehension, and can prevent depression in later life. Exercising can also possibly help in delaying the effects of Alzheimer's disease and other forms of dementia. ${ }^{1}$ In combination with an appropriate diet, it can help to reduce weight. ${ }^{1}$ Regular exercise makes the heart stronger that supports the cardiovascular system to circulate and distribute more oxygen to the body with every heartbeat. It makes the lungs filter healthier and enables the pulmonary system to maximize the amount of oxygen the lungs can take in. ${ }^{4}$

Exercise lowers the blood pressure, slightly decreases the levels of total- and low-density lipoprotein-the bad cholesterol, and increases the level of high-density lipoproteinthe good cholesterol. ${ }^{4}$ In addition, colon cancer and some forms of diabetes are less likely to occur in people who exercise regularly. ${ }^{4}$ Physical activity and exercise in any form is a stress relieving activity. A good health is obligatory for doing a good work. Multiple evidences have shown that people who lead active lifestyle are more liable to live longer and less likely to suffer from illness. Exercise also improves an individual's mental health and overall sense of well-being. ${ }^{5}$

Current trend and lifestyle have made an individual become more inclined toward less physical activity. However, to reverse the current trend of inactivity, countries across the WHO European Region desire to create circumstances for people to keep them engaged in physical activities as a part of their everyday life. Action should be on large scale, coherent, and consistent across different levels of government and different sectors, based on the following key principles ${ }^{1}$ :

1. Promote a life-course and whole-of-society approach.

2. Provide attractive environments so as to encourage active transport and physical activity in daily life.

3. Provide and promote safe environments.

4. Promote integrated, multisectoral, and partnership-based approaches.

5. Use evidence-based strategies.

6. Promote health care settings by encouraging physical activities as a primary care.

7. Create opportunities for social and physical activity for older people so as to hold functional capacity and reduce the onset of persistent disease. ${ }^{1}$

\section{Types of Exercises}

Following are a few types of exercises:

1. Endurance or aerobic: These activities increase breathing and heart rate. They keep the heart, lungs, and circulatory system healthy and improve overall fitness, e.g., brisk walking, jogging, swimming, and biking.

2. Strength or resistance training: These exercises make the muscles stronger, e.g., lifting weights and using a resistance band.

3. Balance: These exercises can make it easier to walk on uneven surfaces and help prevent falls, e.g., to improve balance, Tai chi or exercises like standing on one leg.
4. Flexibility: These exercises stretch the muscles and can help the body stay limber, e.g., yoga and various stretches can make the body more flexible. ${ }^{1}$

The Physical Activity Guidelines for Americans, second edition published by the U.S. Department of Health and Human Services, Office of Disease Prevention and Health Promotion recommended how much physical activity is needed to make us healthy. Following tips are based totally on current clinical evidence helping the connections between physical activity, overall health and well-being, disease prevention, and best of life.

\section{American Heart Association Tips for Adults}

Following are a few tips from the American Heart Association for adults:

1. Get at least $150 \mathrm{~min} / \mathrm{wk}$ of moderate intensity aerobic interest or $75 \mathrm{~min} / \mathrm{wk}$ of vigorous aerobic activity, or a combination of both, preferably spread during the week.

2. Add moderate- to high-intensity muscle-strengthening activity (which includes resistance or weights) on at least $2 \mathrm{~d} / \mathrm{wk}$.

3. Spend less time sitting. Even light-intensity activity can offset some of the risks of being sedentary.

4. Gain even more advantages by being active at least 300 min (5 hours)/wk.

5. Increase the amount and intensity gradually over time. ${ }^{6}$

\section{American Heart Association Guidelines for Youngsters}

Following are the guidelines from the American Heart Association for youngsters:

1. Children 3 to 5 years old must be physically active and should have lots of possibilities to move at some point of the day.

2. Kids 6 to 17 years old should have a minimum of $60 \mathrm{~min} / \mathrm{d}$ of moderate-to vigorous-intensity physical hobby, in most cases aerobic activities. Include an energy-intensive hobby for at least 3 days a week.

3. Include muscle- and bone-strengthening (weight-bearing) activities at least 3 days a week.

4. Increase quantity and intensity step by step over time. ${ }^{6}$

World Health Organization, Europe, in 2020 has prepared hard and fast examples of primarily home-based physical games with the intention to support people with staying physically active while at home. These are as follows:

a. Knee to elbow: Touch one knee with the alternative elbow, changing sides. Find your own pace. Try to carry out this for 1 to 2 minutes, rest for 30 to 60 seconds, and repeat up to five times. This exercise ought to increase your coronary heart and respiration rates. 
b. Plank: Support your forearms firmly on the ground, with the elbows underneath the shoulders. Keep the hips at the level of the head. Hold for 20 to 30 seconds (or extra, if possible), relax for 30 to 60 seconds, and repeat up to five times. This workout strengthens your belly, arms, and legs.

c. Back extensions: Touch your ears along with your fingertips and raise your upper body, keeping the legs on the ground. Lower the upper frame again. Perform this workout 10 to 15 times (or extra), relaxation for 30 to 60 seconds, and repeat up to five times. This exercise strengthens your back muscles.

d. Squats: Place your feet at hip distance with the toes pointing slightly outward. Bend the knees as much as you can without any problem, preserving the heels on the floor and the knees over (no longer in the front of) the toes. Bend and stretch the legs. Perform this exercise for 10 to 15 times (or extra), rest for 30 to 60 seconds, and repeat up to five times. This workout strengthens your legs and gluteus.

e. Side knee lifts: Touch your knee together with your elbow, lifting the knee to the side, alternating facets. Find your own pace. Try to carry out this for 1 to 2 minutes, relax for 30 to 60 seconds, and repeat up to five times. These exercises are meant to increase your heart and respiration rates.

f. Superman: Place your hands under your shoulders and knees below your hips. Lift one arm forward and your alternate leg back, changing sides. Perform this workout for 20 to 30 times (or more), relax for 30 to 60 seconds, and repeat up to five times. This exercise strengthens your belly, gluteus, and hamstring.

g. Bridge: Plant your toes firmly on the floor with the knees over the heels. Lift the hips as a good deal as it feels snug and slowly lower them again. Perform this workout 10 to 15 times (or greater), rest for 30 to 60 seconds, and repeat up to five times. This workout strengthens your gluteus.

h. Chair dips: Hold onto the seat of a chair, along with your toes about half a meter away from the chair. Bend your hands as you lower your hips to the ground, and then straighten the arms. Perform this exercise 10 to 15 times (or greater), relax for 30 to 60 seconds, and repeat up to five times. This exercising strengthens your triceps.

i. Child's pose: With the knees on the floor, carry your hips to your heels. Rest your stomach to your thighs and actively stretch your arms ahead. Breathe normally. Hold this position for 20 to 30 seconds (or extra). This posture stretches your returned shoulders and sides of the body.

j. Seated meditation: Sit without difficulty on the floor together with your legs crossed (alternatively, take a seat on a chair). Make positive your returned is straight. Close your eyes, relax your body, and deepen your respiration step by step. Concentrate on your breath, trying no longer to cognizance on any mind or concerns. Stay in this posture for 5 to 10 minutes or more, to loosen up and clear your mind. k. Legs up the wall: Bring your hips close $(5-10 \mathrm{~cm})$ to the wall and permit rest to your legs. Close your eyes, relax your frame, and deepen your respiration step by step. Concentrate on your breath, trying now not to focus on any thoughts or concerns. Rest in this pose for up to 5 minutes. This position is supposed to be cushy, relaxing, and de-stressing. ${ }^{1}$

\section{Few tips in keeping with the National Institute of Health, 2018, are as follows:}

a. The optimal span of the physical activity on a daily basis or on maximum days is at least 30 minutes.

b. Check with the physician before you start a regular workout application if there are any health conditions, for e.g., coronary heart trouble, excessive blood pressure, diabetes, weight problems, etc., or if age is 40 years or older.

c. When beginning an exercise routine, few may have muscle pain and discomfort at the beginning. If it does, one may be working too difficult and want to ease up. Stop exercising and see the health practitioner if there is any chest ache or discomfort before the next workout session.

d. Ask the doctor for activities that are safe if one has osteoporosis. If one has low bone mass, guard your spine by avoiding sporting events or activities that flex, bend, or twist it.

e. Avoid excessive-effective exercise to lower the chance of breaking a bone if there is any problem associated with bone.

f. Maintain a habit for correct posture. ${ }^{2}$

According to Elmagd physical activity and exercise can have immediate and long-term health benefits. Most importantly, regular activity can improve the quality of life. A minimum of 30 minutes of exercise a day can allow you to enjoy these benefits. $^{7}$

\section{Conclusion}

Exercises enhance not only the physical body but also the all-round health of an individual. It improves the sense of well-being, decreases the risk of developing diseases, viz., heart disease, diabetes, obesity, and cancer. Exercising daily helps in reducing anxiety and stress among the individuals. Exercising daily can further improve the concentration power and sharpen one's memory. In a nutshell, exercise can improve self-confidence and boost the happy hormones, which make an individual happier than anything else.

\section{Conflict of Interest}

None declared.

\section{References}

1 World Health Organization-Regional Office for Europe. 2020. Physical activity to stay healthy. Available at: http:// www.euro.who.int/en/health-topics/disease-prevention/ 
physical-activity/data-and-statistics/physical-activity-tostay-healthy. Accessed October 27, 2020

2 National Institute of Health Osteoporosis and Related Bone Diseases. National Resource Centre. Exercises for your bone health; 2018. Available at: https://www.bones.nih.gov/healthinfo/bone/bone-health/exercise/exercise-your-bonehealth

3 Medline Plus Trusted Health Information for You. Exercises and Physical Fitness. U.S. National Library of Medicine; 2017. Available at: https://medlineplus.gov/exerciseandphysicalfitness.html

4 Johnston BD. Benefits of exercise. MSD manual consumer version; 2018. Available at: https://www.msdmanuals.com/en-pt/ home/fundamentals/exercise-and fitness/benefits-of-exercise? query=Overview\%20of\%20Exercise. Accessed October 27, 2020

5 Skills you need. Helping you develop life skills. The importance of exercise; 2017. Available at: https://www.skillsyouneed. com/ps/exercise.html. Accessed October 27, 2020

6 American Heart Association Recommendations for Physical Activity in Adults and Kids. Available at: https://www.heart. org/en/healthy-living/fitness/fitness-basics/aha-recs-forphysical-activity-in-adults. Accessed October 27, 2020

7 Elmagd MA. Benefits, need and importance of daily exercise. International J Phys Educ Sports Health 2016;3(5):22-27 